Let $x \in E$. Denote by $S(x, \epsilon)$ the sphere of center $x$ and radius $\epsilon$. Denote by $G(x, \epsilon)$ the greatest distance of the points of $\bar{S}(x, \epsilon)$ from $E$. We are going to prove the following theorem.

Theorem 2. For almost all points of $E$ (that is, for all points of $E$ except a set of $n$-dimensional measure 0 )

$$
\lim G(x, \epsilon) / \epsilon=0 .
$$

It is well known that almost all points of $E$ are points of Lebesgue density 1 . Let $x$ be such a point, and suppose that

$$
\lim G(x, \epsilon) / \epsilon \neq 0 .
$$

This means that there exists an infinite sequence $\epsilon_{i}$ and points $z_{i}$, $z_{i} \in \bar{S}\left(x, \epsilon_{i}\right), \epsilon_{i} \rightarrow 0$, such that the distance of $z_{i}$ from $E$ is greater than $c \epsilon_{i}$, where $c>0$. But this clearly means that $x$ can not have Lebesgue density 1 . This contradiction establishes our theorem.

UNIVERSITY OF MICHIGAN

\title{
ON MONOTONE RETRACTABILITY INTO SIMPLE ARCS
}

\section{G. T. WHYBURN}

In recent work on the area of surfaces Rado [1] has had occasion to use the following properties as applied to locally connected continua $A$ :

( $\pi$ ) Every simple arc in $A$ is a monotone retract of $A$;

(II) Every monotone image of $A$ has property ( $\pi$ ).

Radó has noted that (II) implies ( $\pi$ ) and that the sphere and 2-cell each have (II). In this paper it will be shown that (1) for locally connected continua in general, property (II) is equivalent to unicoherence, (2) for plane locally connected continua, property $(\pi)$ is equivalent to unicoherence, and (3) every closed 2-dimensional connected manifold has property $(\pi)$.

To clarify our meaning, we recall that a continuum is compact, connected and metric. A continuous mapping $f(A)=B$ on a continuum $A$ is monotone provided $f^{-1}(y)$ is a continuum for $y \in B$. If 1945.

Presented to the Society, September 17, 1945; received by the editors September 8,

${ }^{1}$ Numbers in brackets refer to the Bibliography at the end of the paper. 
$X \subset A$ and a monotone mapping $f(A)=X$ exists such that $f(x)=x$ for every $x \in X, X$ is a monotone retract of $A$ and $f$ is a monotone retraction of $f$ into $A$.

THEOREM 1. In order that a locally connected continuum $M$ have property (II) it is necessary and sufficient that it be unicoherent.

Proof. If $M$ is unicoherent, any simple arc $a b$ in $M$ is a monotone interior retract of the cyclic chain $C(a, b)$ in $M$ by pp. 212-213 of [2]; and since $C(a, b)$ is a monotone retract of $M$, it follows that a monotone retraction of $M$ onto $C(a, b)$ followed by a monotone retraction of $C(a, b)$ onto $a b$ yields a monotone retraction of $M$ onto $a b$. Thus $M$ has property $(\pi)$. Since unicoherence is invariant under monotone mappings, it follows that $M$ also has property (II). Thus the condition is sufficient.

To show the necessity, suppose $M$ has property (II), but, contrary to our assertion, $M$ is not unicoherent. We shall show that some monotone image of $M$ fails to have property $(\pi)$ and thus arrive at a contradiction. It follows by (3.5) and (3.21), pp. 215-216, of [2] that there exist continua $A, B, H$ and $K$ in $M$ such that $A \cdot B=0, H \cdot K$ $=A+B$, and $H+K=M$. For let $f$ be an interior non-alternating retraction of $M$ onto a simple closed curve $J$ as given by (3.2), (3.5) and (3.21), pp. 214-216, of [2]. Let $x_{1}, x_{2} \in \alpha, y_{1}, y_{2} \in \beta$ be chosen so that the pairs $x_{1}, y_{2}$ and $x_{2}, y_{1}$ separate each other on $J$. Then the continua

$$
\begin{array}{lll}
A=f^{-1}\left(x_{1} y_{1}\right), & B & =f^{-1}\left(x_{2} y_{2}\right), \\
H=f^{-1}\left(y_{1} x_{1} x_{2} y_{2}\right), & & K=f^{-1}\left(x_{1} y_{1} y_{2} x_{2}\right)
\end{array}
$$

satisfy all our requirements.

Now let us decompose $M[3]$ into the sets $A, B$ and single points of $M-(A+B)$. Let $g(M)=M^{\prime}$ be the associated mapping for this decomposition. Then $g$ is monotone. However, if $a^{\prime}=g(A), b^{\prime}=g(B)$, $H^{\prime}=g(H), K^{\prime}=g(K)$ and $a^{\prime} b^{\prime}$ is an arc in $H^{\prime}$ from $a^{\prime}$ to $b^{\prime}$, there can exist no monotone retraction $\phi\left(M^{\prime}\right)=a^{\prime} b^{\prime}$. For if so, $\phi\left(K^{\prime}\right)$ would be connected; and since it would contain $a^{\prime}$ and $b^{\prime}$, it would also contain a point $x^{\prime}$ interior to $a^{\prime} b^{\prime}$. But then $\phi^{-1}\left(x^{\prime}\right) \cdot\left(K^{\prime}-a^{\prime}-b^{\prime}\right) \neq 0$ $\neq \phi^{-1}\left(x^{\prime}\right) \cdot\left(H^{\prime}-a^{\prime}-b^{\prime}\right)$ and $\phi^{-1}\left(x^{\prime}\right) \subset\left(K^{\prime}-a^{\prime}-b^{\prime}\right)+\left(H^{\prime}-a^{\prime}-b^{\prime}\right)$, contrary to the fact that $\phi^{-1}\left(x^{\prime}\right)$ is connected.

THEOREM 2. If $X$ is a compact metric space and $f(X)=Y$ is a monotone retraction, for any component $R$ of $X-Y$ we have

$$
f(\bar{R})=\bar{R}-R .
$$

Proof. Since obviously $f(\bar{R}) \supset f(\bar{R}-R)=\bar{R}-R$ and $R \cdot f(R)=0$, we 
have only to show that $f(R) \subset \bar{R}$. To this end let $y \in f(R)$, let $x \in R \cdot f^{-1}(y)$ and let $C_{x}$ be the component of $f^{-1}(y)-y$ containing $x$. Since $f^{-1}(y)$ is a continuum, $\bar{C}_{x} \supset y$. Also, since $f$ is a retraction we have $Y \cdot f^{-1}(y)=y$ and hence $C_{x} \cdot Y=0$. Thus $C_{x} \subset X-Y$. Hence $C_{x} \subset R$ because $R \supset x$. Accordingly $\bar{R} \supset \bar{C}_{x} \supset y$, as was to be proven.

COROLLARY 1. If $Y$ is a monotone retract of a compact metric space $X$, then for any component $R$ of $X-Y, \bar{R}-R$ is a continuum.

CoRollary 2. If $X$ is a locally connected continuum and $Y$ is any monotone retract of $X$, the boundary of each component of $X-Y$ is a continuum.

THEOREM 3. In order that a plane locally connected continuum have property $(\pi)$ it is necessary and sufficient that it be unicoherent.

The sufficiency results from Theorem 1 , since property (II) implies property $(\pi)$.

To prove the necessity, let $M$ be any plane non-unicoherent locally connected continuum. We shall show that $M$ cannot have property $(\pi)$. Now $E$ has at least one non-unicoherent true cyclic element $E$ and it will suffice to show that $E$ contains a simple arc $\alpha$ which is not a monotone retract of $E$. For if $M$ could be retracted onto $\alpha$ by a monotone retraction $g(M)=\alpha, g$ would be monotone on $E$ and hence would retract $E$ monotonically onto $\alpha$.

Now as $E$ is cyclic and non-unicoherent, it contains simple closed curves $J$ and $C$ such that $J$ is the boundary of the unbounded comcomplementary domain $U$ of $E$ and $C$ is the boundary of a bounded complementary domain $R$ of $E$. We distinguish three cases.

1. If $J \cdot C=0$, then since $E$ is cyclic it contains disjoint arcs $a_{1} b_{1}$ and $a_{2} b_{2}$ such that $a_{i} b_{i} \cdot J=a_{i}$ and $a_{i} b_{i} \cdot c=b_{i}, i=1,2$. Then if $b_{1} \times b_{2}$ and $b_{1} y b_{2}$ are the two arcs of $C$ from $b_{1}$ to $b_{2}$, the $\operatorname{arc} \alpha=a_{1} b_{1}+b_{1} x b_{2}$ $+b_{2} a_{2}$ cannot be a monotone retract of $E$. For if $Q$ is the component of $E-\alpha$ containing $b_{1} y b_{2}-\left(b_{1}+b_{2}\right)$, the boundary of $Q$ is not connected because it contains $b_{1}$ and $b_{2}$ but does not contain $x$. Hence, by Corollary 2, $\alpha$ is not a monotone retract of $E$.

2. If $J \cdot C$ is a single point $p$, there is an arc $a b$ in $E-p$ with $a b \cdot J=a$ and $a b \cdot C=b$. Then if $b x p$ and $b y p$ are the two arcs of $C$ from $b$ to $p$, the arc $\alpha=a b+b x p$ cannot be a monotone retract of $E$, because again the boundary of the component $Q$ of $E-\alpha$ containing byp $-(a+p)$ is not connected.

3. If $J \cdot C$ contains two distinct points $p$ and $q$, and $p x q$ and $p y q$ are the two arcs of $C$ from $p$ to $q$, then the arc $\alpha=p x q$ cannot be a 
monotone retract of $E$ because the boundary of the component $Q$ of $E-\alpha$ containing $p y q-(p+q)$ is not connected.

THEOREM 4. Any closed 2-dimensional connected manifold has prop$\operatorname{erty}(\pi)$.

For let $M$ be such a manifold and let $\alpha$ be any simple arc on $M$. There exists a 2-cell $E$ on $M$ containing $\alpha$ and such that if $J$ is the edge of $E$, then $J \cdot \alpha=a$ where $\alpha=a b$. There exists a monotone retraction $g(E)=\alpha$ such that $g(J)=a$. For we may suppose $E$ to be the set $|z| \leqq 1$ and $\alpha$ the set $y=0,0 \leqq x \leqq 1$ in a complex plane; and the mapping $g: w=|z|$, with the $w$-plane identical with the $z$-plane, meets our requirements. Then if we define $g(p)=a$ for all $p \in M-E$, clearly $g$ retracts $M$ monotonically onto $\alpha$.

\section{BIBLIOGRAPHY}

1. T. Rad6, On continuous mappings of Peano spaces, Trans. Amer. Math. Soc. vol. 58 (1945) pp. 420-454.

2. G. T. Whyburn, Analytic topology, Amer. Math. Soc. Colloquium Publications, vol. 28, New York, 1942.

3. R. L. Moore, Concerning upper semi-continuous collections of continua, Trans. Amer. Math. Soc. vol. 27 (1925) pp. 416-428.

UNIVERSITY OF VIRGINIA 